\title{
Immunonkologie:
}

\section{Neue Therapieoption mit großem Potenzial}

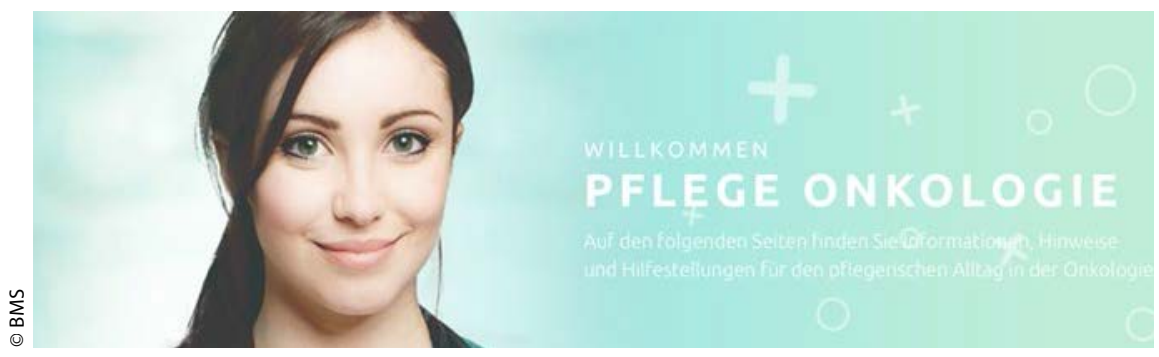

— Fortschritte in der Krebsforschung haben zu deutlich verbesserten Behandlungsmöglichkeiten von Tumor-Erkrankungen geführt. Mit immunonkologischen Therapien, die die natürliche Fähigkeit des körpereigenen $\mathrm{Ab}$ wehrsystems zur Erkennung und Bekämpfung von Tumoren nutzen, konnte das Therapie-Spektrum um eine weitere effektive Option erweitert werden. Konventionelle Krebs-Therapien wirken direkt auf den Tumor bzw. die Tumorzelle. Immunonkologische Wirkstoffe greifen dagegen nicht den Tumor selbst an, sondern bekämpfen Krebszellen indirekt über eine Aktivierung des Immunsystems (Borghaei $\mathrm{H}$ et el. Eur J Pharmacol 2009; 625: 41-54).
Immunonkologische Substanzen überwinden Abwehrstrategien des Tumors: Normalerweise erkennt und zerstört das Immunsystem auch Tumorzellen. Diese nutzen oft jedoch komplexe Strategien - so genannte "Tumor-Escape-Mechanismen" -, um dem Immunsystem zu entkommen (Dunn GP et al. Immunity 2004; 21: 137-148). Diese Erkenntnis hat zur Entwicklung von Immun-Checkpoint-Hemmern als einer der wichtigsten, derzeit zugelassenen immunonkologischen Behandlungsstrategien geführt. Checkpoint-Hemmer überwinden die Escape-Mechanismen des Tumors und schalten das Immunsystem wieder scharf. Auch die Aktivierung natürlicher Killerzel- len zur Bekämpfung von Tumorzellen ist ein effektives immunonkologisches Therapieprinzip.

Grundlegend andere Nebenwirkungen: Da die neuen Medikamente über eine Aktivierung des körpereigenen Abwehrsystems wirken, unterscheidet sich ihr Nebenwirkungsspektrum grundlegend von dem herkömmlicher Therapien wie beispielsweise der Chemotherapie (Overkamp F. Deutsches Ärzteblatt. DOI: 10.3238/PersOnko/2016.02.12.10). Im Vordergrund stehen so genannte immunvermittelte Nebenwirkungen, die sich häufig mit überschießenden Entzündungen präsentieren. Beim Auftreten immunvermittelter Nebenwirkungen muss unverzüglich ein spezialisierter Facharzt kontaktiert werden. Daher ist es wichtig, dass onkologische Pflegekräfte für die entsprechende Symptomatik sensibilisiert sind und diese frühzeitig erkennen.

Wenn Sie mehr zum Wirkprinzip der Immunonkologie oder über das Management immunvermittelter Nebenwirkungen wissen wollen, laden wir Sie herzlich zu einem Besuch unseres Informations-Portals www. pflege-onkologie.de ein. Im Kapitel „Krebs- $\overline{0}$ therapie" finden Sie unter der Rubrik "Im- $\stackrel{\infty}{\frac{\circ}{2}}$ munonkologie" alle wichtigen Informatio- $\frac{\partial}{\tilde{\nu}}$ nen zu dieser neuen Therapieform. (reck) 으

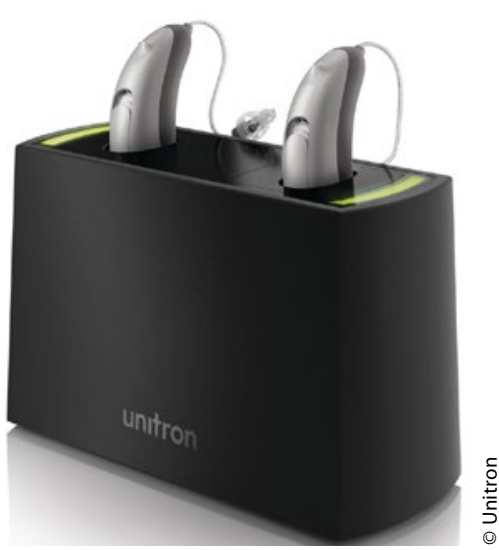

ermöglicht ein besonders natürliches und realistisches Hören, selbst wenn es im Hintergrund, beispielsweise in beruflichen Meetings, beim Einkaufen oder beim Zusammensein mit Freunden, laut zugehen sollte - ein echter Mehrwert im Alltag.

www.unitron.com/de 